\title{
High-power thulium lasers on a silicon photonics platform
}

\author{
Nanxi Li, $, 1,2,{ }^{*}$ Purnawirman, ${ }^{1}$ Zhan Su, ${ }^{1}$ E. Salih Magden, ${ }^{1}$ Patrick T. \\ Callahan, ${ }^{1}$ Katia Shtyrkova, ${ }^{1}$ Ming XIN, ${ }^{1}$ Alfonso Ruocco, ${ }^{1}$ Christopher \\ BAIOCCO, ${ }^{3}$ ERICH P. IPPEN, ${ }^{1}$ FrAnz X. KÄRTNER, ${ }^{1,4}$ JonATHAN D. B. BradLey, ${ }^{1,5}$ \\ Diedrik Vermeulen, ${ }^{1}$ And Michael R. WAtts ${ }^{1}$
}

\author{
${ }^{1}$ Department of Electrical Engineering and Research Laboratory of Electronics, Massachusetts Institute of Technology, 77 Massachusetts \\ Avenue, Cambridge, Massachusetts 02139, USA \\ 2John A. Paulson School of Engineering and Applied Sciences, Harvard University, 29 Oxford Street, Cambridge, Massachusetts, 02138, USA \\ ${ }^{3}$ College of Nanoscale Science and Engineering, State University of New York, 257 Fuller Road, Albany, New York 12203, USA \\ ${ }^{4}$ Center for Free-Electron Laser Science, DESY and Hamburg University, Notkestraße 85, 22607 Hamburg, Germany \\ ${ }^{5}$ Current address: Department of Engineering Physics, McMaster University, 1280 Main Street West, Hamilton, Ontario L8S 4L7, Canada \\ *Corresponding author: nanxili@mit.edu
}

Received XX Month XXXX; revised XX Month, XXXX; accepted XX Month XXXX; posted XX Month XXXX (Doc. ID XXXXX); published XX Month XXXX

\begin{abstract}
Mid-infrared laser sources are of great interest for various applications, including Light Detection and Ranging (LIDAR), spectroscopy, communication, tracegas detection, and medical sensing. Silicon photonics is a promising platform that enables these applications to be integrated on a single chip with low cost and compact size. Silicon-based high-power lasers have been demonstrated at $1.55 \mu \mathrm{m}$ wavelength, while in the $2 \mu \mathrm{m}$ region, to the best of our knowledge, high-power, highefficiency and monolithic light sources have been minimally investigated. In this letter, we report on highpower CMOS-compatible thulium-doped distributed feedback and distributed Bragg reflector lasers with single-mode output powers up to $267 \mathrm{~mW}$ and $387 \mathrm{~mW}$, and slope efficiencies of $14 \%$ and $23 \%$, respectively. More than $70 \mathrm{~dB}$ side-mode suppression ratio is achieved for both lasers. This work extends the applicability of silicon photonic microsystems in the 2 $\mu \mathrm{m}$ region. (C) 2017 Optical Society of America
\end{abstract}

OCIS codes: (130.3120) Integrated optics devices; (140.0140) Lasers and laser optics; (230.5750) Resonators.

http://dx.doi.org/10.1364/OL.99.099999

Laser sources in the $2 \mu \mathrm{m}$ wavelength region have many applications such as LIDAR, spectroscopy, optical waveform generation and synthesis, material processing, communication, and trace-gas detection systems. The high water absorption at $2 \mu \mathrm{m}$ also makes such lasers good candidates for medical applications [1]. Furthermore, $2 \mu \mathrm{m}$ wavelength lasers in pulsed mode could be used as pumps for nonlinear processes in silicon such as mid-IR optical parametric amplification (OPA) [2]. Silicon is the material of choice for both microelectronic circuits and integrated photonics components around $1.31 \mu \mathrm{m}$ and $1.55 \mu \mathrm{m}$. To extend the applicability of silicon photonics microsystems to the $2 \mu \mathrm{m}$ wavelength region, complementary metal-oxide semiconductor (CMOS) compatible integrated laser sources around $2 \mu \mathrm{m}$ are critical. These lasers will enable highly compact devices that can perform the task of current complex bulky laboratory setups.

Among the methods to integrate lasers on a silicon platform [3-6], deposition of rare-earth-doped $\mathrm{Al}_{2} \mathrm{O}_{3}$ glass has proven to be effective due to several advantages. Firstly, rare-earth-doped $\mathrm{Al}_{2} \mathrm{O}_{3}$ glass can be deposited as a single-step back-end-of-line process on silicon wafers without requiring any additional etch steps [7-10]. Secondly, common rare-earth materials such as erbium and thulium have a wide emission spectrum, which enables wavelength tunability and design flexibility [11-13]. Thirdly, the rare-earth doped gain media do not involve free carriers as semiconductor lasers do, enabling low losses and lasers with narrow linewidths [14-16]. Lastly, the low thermooptic effect of the host medium enables operation over a wide temperature range [17]. The state-of-the-art for high-power lasers fabricated using this methodology was demonstrated to be $75 \mathrm{~mW}$ continuous-wave (CW) output power in an erbiumdoped waveguide laser operating around $1.55 \mu \mathrm{m}$ [18]. Among rare-earth elements, thulium is particularly attractive for integrated lasers on silicon since its emission spectrum is around $2 \mu \mathrm{m}$. Integrated thulium-doped channel waveguide lasers on tungstate were reported with high power and slope efficiencies [19, 20]. However, so far work on thulium-doped high-power lasers on a CMOS-compatible platform has been absent.

Prior to this work, we have reported a thulium-doped microcavity laser with sub-milliwatt output power and multi- 
mode operation [21, 22]. However, high output power and single-mode operation are desired for many applications. In this paper, we demonstrate high-power thulium-doped waveguide lasers that were fabricated on silicon chips in a 300-mm CMOS

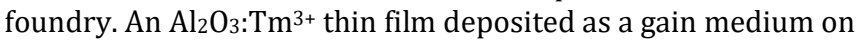
top of a buried $\mathrm{Si}_{3} \mathrm{~N}_{4}$ strip in $\mathrm{SiO}_{2}$ acts as a rib waveguide on a silicon substrate. The narrow reflection bandwidths of distributed feedback (DFB) and distributed Bragg reflector (DBR) structures enable single-mode output. The maximum on-chip lasing power achieved for the DFB and DBR devices were $267 \mathrm{~mW}$ and $387 \mathrm{~mW}$, with slope efficiencies of $14 \%$ and $23 \%$, respectively.

The waveguide cross-section of the DFB laser is shown in Fig. 1(a). The width and separation of the $\mathrm{Si}_{3} \mathrm{~N}_{4}$ bars are

(a)

(b)

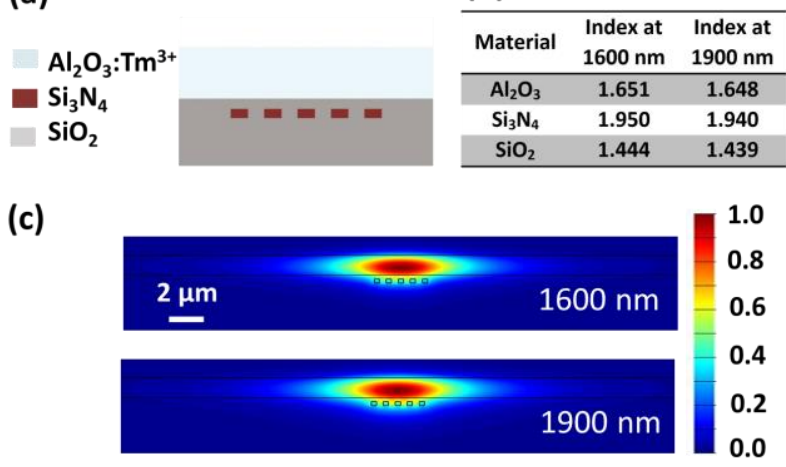

(d)

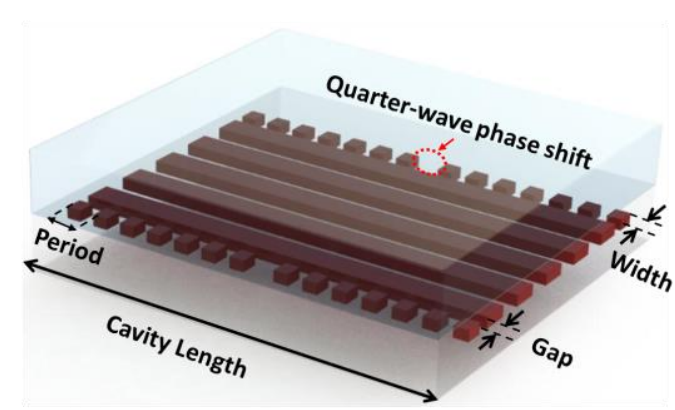

(e)

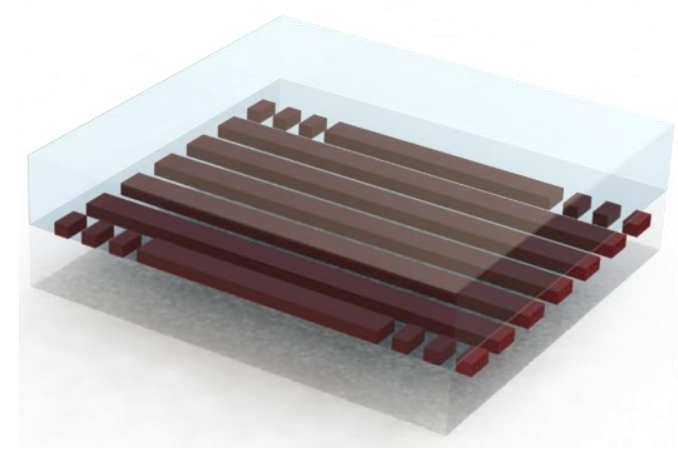

Fig. 1 Integrated thulium DFB and DBR laser designs. (a) Cross-section of the laser gain waveguide including five strips of $\mathrm{Si}_{3} \mathrm{~N}_{4}$. (b) Refractive indices of the waveguide materials at both pump and signal wavelengths. (c) Fundamental TE field intensity for the pump (1600 nm) and laser output (1900 nm) wavelengths in the DFB waveguide. 3D illustrations of (d) the DFB laser and (e) the DBR laser, showing the different material layers and cavity features (not to scale).

optimized to be $300 \mathrm{~nm}$ and $350 \mathrm{~nm}$ respectively to provide high mode confinements for both pump and signal modes within the $\mathrm{Al}_{2} \mathrm{O}_{3}: \mathrm{Tm}^{3+}$ film. The confinement factors within the thulium-doped gain region for pump and signal are calculated to be $90 \%$ and $85 \%$ respectively using a finite-difference 2D mode solver. The oxide gap between the $\mathrm{Si}_{3} \mathrm{~N}_{4}$ layer and the $\mathrm{Al}_{2} \mathrm{O}_{3}$ layer is $200 \mathrm{~nm}$. The refractive indices of the materials used in our laser at both pump and signal wavelengths are listed in Fig. 1(b). The transverse-electric (TE) field intensity of the fundamental mode is shown in Fig. 1(c). For the DBR laser, the gain waveguide cross section has seven $\mathrm{Si}_{3} \mathrm{~N}_{4}$ bars instead of five. Gratings are added on both sides of the gain waveguide. We designed DFB and DBR cavities for different laser wavelengths by varying the grating periods.

Perspective views of the DFB and DBR lasers are illustrated in Fig. 1(d) and (e) respectively. For the DFB laser, the lateral gap between the grating and gain waveguide is designed to be $450 \mathrm{~nm}$, and the grating width is chosen to be $260 \mathrm{~nm}$ in order to provide enough feedback at the designed laser wavelength. The coupling coefficient $(\kappa)$ is calculated to be $1.0 \times 10^{3} \mathrm{~m}^{-1}$. For the DBR laser, the grating width and gap follow the pattern of seven $\mathrm{Si}_{3} \mathrm{~N}_{4}$ strips of gain waveguide between two DBR mirrors, with coupling coefficient $(\kappa)$ of $1.3 \times 10^{3} \mathrm{~m}^{-1}$. The cavity lengths for both lasers are $2 \mathrm{~cm}$, limited by the maximum length of the chip. For a laser cavity length shorter than $2 \mathrm{~cm}$, with the same

(a)

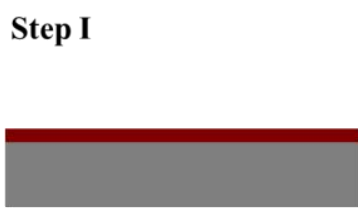

Step III

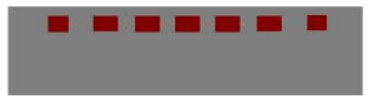

(b)

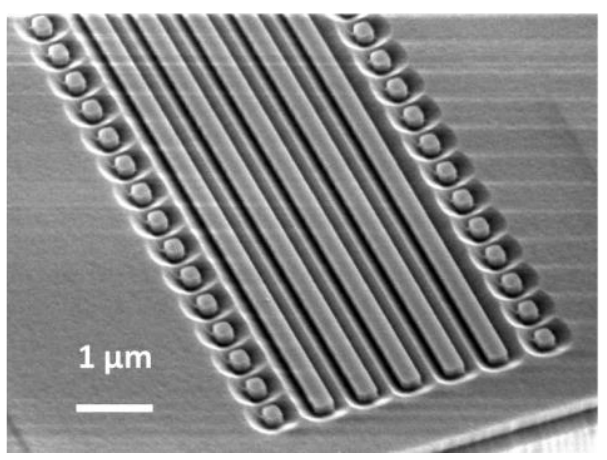

Fig. 2 (a) Laser fabrication steps: (I) deposition of $\mathrm{Si}_{3} \mathrm{~N}_{4}$ layer via PECVD; (II) pattering of $\mathrm{Si}_{3} \mathrm{~N}_{4}$ layer yielding 5-segment (DFB) or seven-segment (DBR) waveguides in the center for mode confinement and additional periodic segments at each side for distributed feedback; (III) deposition of $\mathrm{SiO}_{2}$ followed by top surface planarization; (IV) deposition of the thulium-doped $\mathrm{Al}_{2} \mathrm{O}_{3}$ gain medium by reactive co-sputtering. (b) SEM image of the $\mathrm{Si}_{3} \mathrm{~N}_{4}$ pattern after HF etching of the SiO2 top cladding from Step III. 
pump power, the lasing power decreases. The coupling coefficient and cavity length product $(\kappa \cdot L)$ for DFB and DBR are 20 and 26 respectively. By characterizing the cavity response of the DBR laser, the reflectivity of the grating on each side is estimated to be $70 \%$. Such grating reflectivity enables the laser to achieve a few hundred $\mathrm{mW}$ of output power, while still maintain relatively high $Q$ of the DBR cavity. The effective refractive index ( $n_{\text {eff }}$ ) of the waveguide is calculated using a finite-difference 2D mode solver, considering the grating as perturbations on both sides of the segmented $\mathrm{Si}_{3} \mathrm{~N}_{4}$-rib to provide feedback. The $n_{\text {eff }}$ is calculated to be 1.565 . The grating period $(\Lambda)$ can be obtained using the following equation:

$$
\Lambda=\frac{\lambda}{2 n_{\text {eff }}}
$$

where $\lambda$ is the designed laser wavelength.

The lasers were fabricated in a state-of-the-art CMOS foundry on a $300-\mathrm{mm}$ silicon wafer. The fabrication process is illustrated in Fig. 2 (a). The $\mathrm{Si}_{3} \mathrm{~N}_{4}$ layer is deposited on top of a $\mathrm{SiO}_{2}$ layer, both via plasma-enhanced chemical-vapor deposition (PECVD), followed by a surface polishing process to reduce optical scattering loss. Then the $\mathrm{Si}_{3} \mathrm{~N}_{4}$ layer is patterned using 193-nm immersion lithography and reactive ion etching, as shown in step II. Next, in step III, we deposit a $\mathrm{SiO}_{2}$ layer and planarize the top surface via chemical-mechanical polishing (CMP). The thickness of the $\mathrm{SiO}_{2}$ layer on the $\mathrm{Si}_{3} \mathrm{~N}_{4}$ layer is $200 \mathrm{~nm}$. After that, the $\mathrm{Al}_{2} \mathrm{O}_{3}: \mathrm{Tm}^{3+}$ film is deposited at a thickness of $1100 \mathrm{~nm}$ via reactive co-sputtering as shown in step IV. The substrate temperature is measured to be $350{ }^{\circ} \mathrm{C}$ using a thermocouple directly in contact with chip. The background loss of the $\mathrm{Al}_{2} \mathrm{O}_{3}: \mathrm{Tm}^{3+}$ film is measured to be $<0.1 \mathrm{~dB} / \mathrm{cm}$. Fabrication runs with different doping levels revealed an optimum $\mathrm{Tm}^{3+}$ doping concentration of $3.0 \times 10^{20} \mathrm{~cm}^{-3}$. At the same pump power, but lower doping concentration, the lasing power of the device decreases due to lower gain; while for higher doping

(a)

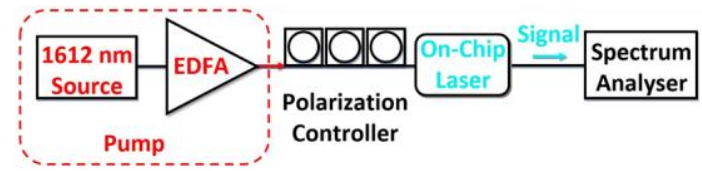

(b)

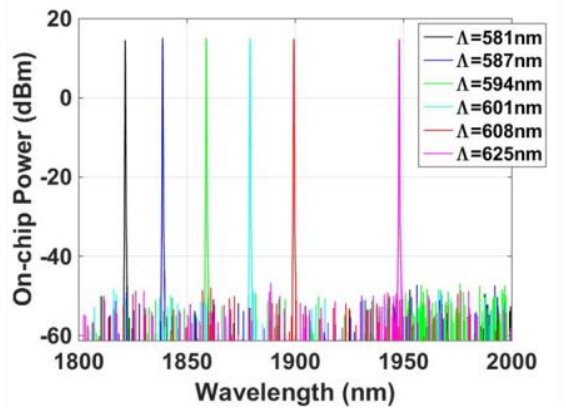

Fig. 3 (a) Measurement setup: a $1612 \mathrm{~nm}$ laser source together with a high-power EDFA, followed by a polarization controller to ensure that the fundamental TE mode is coupled into the gain waveguide of the laser. An optical spectrum analyser is used to capture the spectrum; (b) Output spectrum of DFB lasers with different grating periods, showing single-mode lasing at $1820 \mathrm{~nm}$, $1840 \mathrm{~nm}, 1860 \mathrm{~nm}, 1880 \mathrm{~nm}, 1900 \mathrm{~nm}$, and $1950 \mathrm{~nm}$. concentration, the lasing power of the device decreases due to doped-ion clustering or quenching $[18,23,24]$. In order to visualize the $\mathrm{Si}_{3} \mathrm{~N}_{4}$ pattern, after step III and before step IV, 140s hydrogen fluoride (HF) etching is used to remove the $\mathrm{SiO}_{2}$ top cladding. A scanning electron microscope (SEM) image of the gain waveguide and side gratings is shown in Fig. 2(b).

The measurement setup for characterizing the lasers is illustrated in Fig. 3(a). A laser source at $1612 \mathrm{~nm}$ together with an L-band Erbium-doped fiber amplifier (EDFA) were used for optical pumping. A polarization controller was used to ensure that the pump light was coupled into the fundamental TE mode of the gain waveguide. A cleaved single-mode SMF-28 fiber was used to butt-couple the pump light onto the chip, and another cleaved single-mode SM-2000 fiber was used to butt-couple the output signal of the laser from the chip. The fiber-to-chip coupling losses were measured to be $7.1 \mathrm{~dB}$ for SMF-28 at the pump wavelength, and $7.9 \mathrm{~dB}$ for SM-2000 at the laser output wavelength. The output signal was coupled into an optical spectrum analyzer (Yokogawa AQ6375) to capture the spectrum. The grating period design variations are $581 \mathrm{~nm}, 587 \mathrm{~nm}$, $594 \mathrm{~nm}, 601 \mathrm{~nm}, 608 \mathrm{~nm}$ and $625 \mathrm{~nm}$. They were calculated using equation (1), with corresponding wavelengths at $1820 \mathrm{~nm}$, $1840 \mathrm{~nm}, 1860 \mathrm{~nm}, 1880 \mathrm{~nm}, 1900 \mathrm{~nm}$ and $1950 \mathrm{~nm}$ respectively. The measured optical spectra of the corresponding DFB laser designs are shown in Fig. 3(b). Within the broad gain bandwidth of the $\mathrm{Al}_{2} \mathrm{O}_{3}: \mathrm{Tm}^{3+}$, we are able to precisely control the laser wavelength by choosing the proper grating period.

From the DFB and DBR laser grating period design variations, we selected the devices that operate near the peak of the thulium emission spectrum for the lasing slope efficiency measurements. The slope efficiency curves for DFB and DBR lasers are shown in Fig. 4 (a) and (b) respectively. The maximum on-chip laser output power of the DFB and DBR lasers were measured to be $267 \mathrm{~mW}$ and $387 \mathrm{~mW}$, respectively. The powers (a)

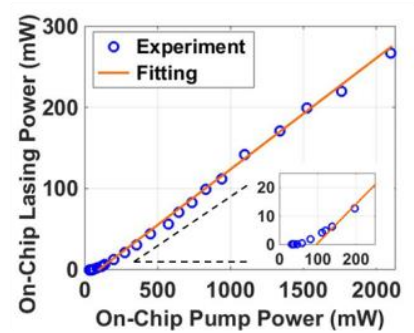

(c)

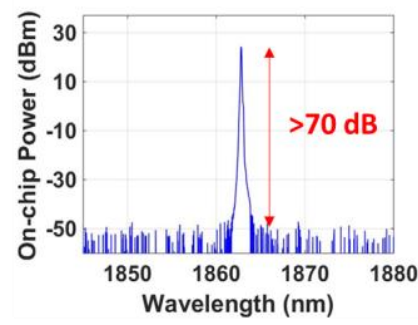

(b)

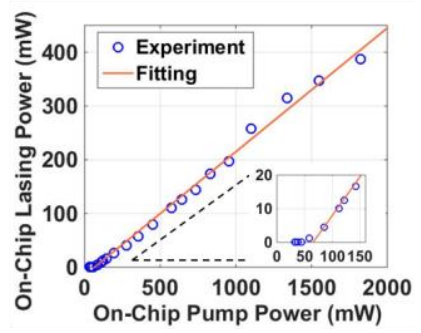

(d)

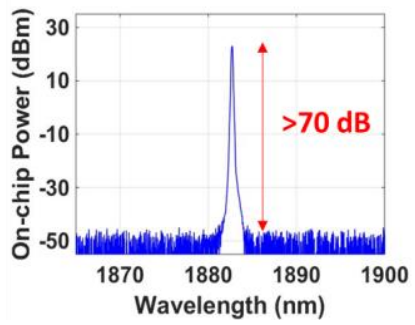

Fig. 4 (a) DFB and (b) DBR laser output curves, showing 14\% and $23 \%$ slope efficiency, $267 \mathrm{~mW}$ and $387 \mathrm{~mW}$ maximum output power, and $96 \mathrm{~mW}$ and $65 \mathrm{~mW}$ lasing threshold, respectively. The output spectra of (c) DFB and (d) DBR lasers at $1861 \mathrm{~nm}$ and $1881 \mathrm{~nm}$, respectively, obtained with up to $2 \mathrm{~W}$ on-chip pump power and showing side-mode suppression ratios $>70 \mathrm{~dB}$. 
were measured from a single output end of the lasers, and the fiber coupling losses are calibrated out. An equivalent level of power could be obtained when pump is launched from the other side of the laser, considering the symmetry of the cavity. Using linear curve fitting, the single-sided slope efficiencies for the DFB and DBR lasers were found to be $14 \%$ and $23 \%$, with laser thresholds of $96 \mathrm{~mW}$ and $65 \mathrm{~mW}$ at 1612-nm pumping. The peak laser wavelengths with up to $2 \mathrm{~W}$ on-chip pump power for the DFB and DBR lasers were recorded by the spectrum analyzer, as shown in Fig. 4(c) and (d) respectively. Single-mode operation was enabled by the narrow bandwidth grating. The side-mode suppression ratio reached more than $70 \mathrm{~dB}$.

In summary, we have designed, fabricated and characterized highpower thulium DFB and DBR lasers integrated on a silicon chip. A CMOS-compatible segmented $\mathrm{Si}_{3} \mathrm{~N}_{4}$ rib-waveguide was used to form the laser cavity. Gratings were added on both sides of the segmented $\mathrm{Si}_{3} \mathrm{~N}_{4}$ rib waveguide to provide feedback and thulium-doped $\mathrm{Al}_{2} \mathrm{O}_{3}$ glass was used as the gain medium. By varying the grating period, single-mode lasers with wavelengths from $1800 \mathrm{~nm}$ to $1950 \mathrm{~nm}$ have been demonstrated within the thulium gain bandwidth. The highest DFB and DBR output powers of $267 \mathrm{~mW}$ and $387 \mathrm{~mW}$ under $1612 \mathrm{~nm}$ pumping were measured at $1861 \mathrm{~nm}$ and $1881 \mathrm{~nm}$ wavelengths, with slope efficiencies of $14 \%$ and $23 \%$, respectively. More than $70 \mathrm{~dB}$ side-mode suppression, which enables high signal-to-noise ratio for chip-scale communication and spectroscopic applications, was observed.

Funding. Defense Advanced Research Projects Agency (DARPA) Direct On-Chip Digital Optical Synthesizer (DODOS) project (HR0011-15-C0056; program manager: Dr. Robert Lutwak). N. Li is sponsored by National Science Scholarship (NSS) from the Agency of Science, Technology and Research (A*STAR), Singapore. K. Shtyrkova is sponsored by National Defense Science and Engineering Graduate Fellowship (32 CFR 168a).

Acknowledgment. The authors would like to acknowledge Professor Federico Capasso for helpful discussion, Gary Riggott and Kurt Broderick for assistance on thulium doped thin film deposition, and Di Zhu for assistance with SEM imaging.

\section{References}

1. S. L. K. Scholle, P. Koopmann, P. Fuhrberg, in Proceedings of Frontiers in Guided Wave Optics and Optoelectronics (2010), p. 471-500.

2. X. Liu, R. M. Osgood, Y. A. Vlasov, and M. J. GreenWilliam, Nat. Photonics, 4, 557-560 (2010).

3. A. W. Fang, E. Lively, Y.-H. Kuo, D. Liang, and J. E. Bowers, Opt. Express, 16, 4413-4419 (2008).
4. H. Rong, R. Jones, A. Liu, O. Cohen, D. Hak, A. Fang, and M. Paniccia, Nature, 433, 725-728 (2005).

5. J. Liu, X. Sun, R. Camacho-Aguilera, L. C. Kimerling, and J. Michel, Opt. Lett., 35, 679-681 (2010).

6. G. Singh, Purnawirman, J. D. B. Bradley, N. Li, E. S. Magden, M. Moresco, T. N. Adam, G. Leake, D. Coolbaugh, and M. R. Watts, Opt. Lett., 41, 1189-1192 (2016).

7. K. Wörhoff, J. D. B. Bradley, F. Ay, D. Geskus, T. P. Blauwendraat, and M. Pollnau, IEEE J. of Quantum Electron., 45, 454-461 (2009).

8. E. S. Magden, Purnawirman, N. Li, G. Singh, J. D. B. Bradley, G. S. Petrich, G. Leake, D. D. Coolbaugh, M. R. Watts, and L. A. Kolodziejski, in CLEO: Science and Innovations (2016), SM1G.2.

9. M. Belt and D. J. Blumenthal, Opt. Express, 22, 10655-10660 (2014).

10. Z. Su, J. D. B. Bradley, N. Li, E. S. Magden, P. Purnawirman, D. Coleman, N. Fahrenkopf, C. Baiocco, T. N. Adam, G. Leake, D. Coolbaugh, D. Vermeulen, and M. R. Watts, in Advanced Photonics 2016 (IPR, NOMA, Sensors, Networks, SPPCom, SOF), IW1A.3.

11. S. Li, D. Zhang, J. Zhao, Q. Yang, X. Xiao, S. Hu, L. Wang, M. Li, X. Tang, Y. Qiu, M. Luo, and S. Yu, Opt. Express, 24, 6341-6349 (2016).

12. N. Li, E. Timurdogan, C. V. Poulton, M. Byrd, E. S. Magden, Z. Su, Purnawirman, G. Leake, D. D. Coolbaugh, D. Vermeulen, and M. R. Watts, Opt. Express, 24, 22741-22748 (2016).

13. Y. Liu, K. Wu, N. Li, L. Lan, S. Yoo, X. Wu, P. P. Shum, S. Zeng, and X. Tan, J. of the Optical Society of Korea, 17, 357-361 (2013).

14. Y. W. Song, S. A. Havstad, D. Starodubov, Y. Xie, A. E. Willner, and J. Feinberg, IEEE Photon. Tech. Lett., 13, 1167-1169 (2001).

15. E. H. Bernhardi, H. A. G. M. van Wolferen, L. Agazzi, M. R. H. Khan, C. G. H. Roeloffzen, K. Wörhoff, M. Pollnau, and R. M. de Ridder, Opt. Lett., 35, 2394-2396 (2010).

16. M. Belt, T. Huffman, M. L. Davenport, W. Li, J. S. Barton, and D. J. Blumenthal, Opt. Lett., 38, 4825-4828, (2013).

17. M. Belt and D. J. Blumenthal, in Optical Fiber Communications Conference and Exhibition (OFC) (2015), pp. 1-3.

18. E. S. Hosseini, Purnawirman, J. D. B. Bradley, J. Sun, G. Leake, T. N. Adam, D. D. Coolbaugh, and M. R. Watts, Opt. Lett., 39, 3106-3109 (2014).

19. K. van Dalfsen, S. Aravazhi, C. Grivas, S. M. García-Blanco, and M. Pollnau, Opt. Lett., 37, 887-889 (2012).

20. K. van Dalfsen, S. Aravazhi, C. Grivas, S. M. García-Blanco, and M. Pollnau, Opt. Lett., 39, 4380-4383 (2014).

21. Z. Su, N. Li, E. Salih Magden, M. Byrd, Purnawirman, T. N. Adam, G. Leake, D. Coolbaugh, J. D. B. Bradley, and M. R. Watts, Opt. Lett., 41, 5708-5711 (2016).

22. J. D. B. Bradley, Z. Su, E. S. Magden, N. Li, M. Byrd, Purnawirman, T. N. Adam, G. Leake, D. Coolbaugh, M. R. Watts, in Proc. SPIE 9744, Optical Components and Materials XIII, $97440 \mathrm{U}$ (February 24, 2016)

23. N. Li, Purnawirman, J. D. B. Bradley, G. Singh, E. S. Magden, J. Sun, and M. R. Watts, in Optoelectronics Global Conference (OGC) (2015), p. 1-2

24. L. Agazzi, K. Wörhoff, and M. Pollnau, The J. of Physical Chemistry C, 117, 6759-6776, (2013). 


\section{Full References}

[1] S. L. K. Scholle, P. Koopmann, P. Fuhrberg "2 um Laser Sources and Their Possible Applications," in Frontiers in Guided Wave Optics and Optoelectronics, B. Pal, Ed., ed: InTech, 2010, pp. 471500.

[2] X. Liu, R. M. Osgood, Y. A. Vlasov, and M. J. GreenWilliam, "Midinfrared optical parametric amplifier using silicon nanophotonic waveguides," Nat Photon, vol. 4, pp. 557-560, 2010.

[3] A. W. Fang, E. Lively, Y.-H. Kuo, D. Liang, and J. E. Bowers, "A distributed feedback silicon evanescent laser," Optics Express, vol. 16, pp. 4413-4419, 2008/03/31 2008.

[4] H. Rong, R. Jones, A. Liu, O. Cohen, D. Hak, A. Fang, and M. Paniccia, "A continuous-wave Raman silicon laser," Nature, vol. 433, pp. 725-728, 2005.

[5] J. Liu, X. Sun, R. Camacho-Aguilera, L. C. Kimerling, and J. Michel, "Ge-on-Si laser operating at room temperature," Optics Letters, vol. 35, pp. 679-681, 2010/03/01 2010.

[6] G. Singh, Purnawirman, J. D. B. Bradley, N. Li, E. S. Magden, M. Moresco, T. N. Adam, G. Leake, D. Coolbaugh, and M. R. Watts, "Resonant pumped erbium-doped waveguide lasers using distributed Bragg reflector cavities," Optics Letters, vol. 41, pp. 1189-1192, 2016/03/15 2016.

[7] K. Worhoff, J. D. B. Bradley, F. Ay, D. Geskus, T. P. Blauwendraat, and M. Pollnau, "Reliable Low-Cost Fabrication of Low-Loss Al2O3:Er Waveguides With 5.4-dB Optical Gain," IEEE Journal of Quantum Electronics, vol. 45, pp. 454-461, 2009.

[8] E. S. Magden, Purnawirman, N. Li, G. Singh, J. D. B. Bradley, G. S. Petrich, G. Leake, D. D. Coolbaugh, M. R. Watts, and L. A. Kolodziejski, "Fully CMOS-Compatible Integrated Distributed Feedback Laser with 250 oC Fabricated Al2O3:Er3+ Gain Medium," in CLEO: Science and Innovations, 2016, p. SM1G.2.

[9] M. Belt and D. J. Blumenthal, "Erbium-doped waveguide DBR and DFB laser arrays integrated within an ultra-low-loss Si3N4 platform," Optics Express, vol. 22, pp. 10655-10660, 2014/05/05 2014.

[10] Z. Su, J. Bradley, N. Li, E. S. Magden, P. Purnawirman, D. Coleman, N. Fahrenkopf, C. Baiocco, T. Adam, G. Leake, D. Coolbaugh, D. Vermeulen, and M. R. Watts, "Ultra-Compact CMOS-Compatible Ytterbium Microlaser," in Advanced Photonics 2016 (IPR, NOMA, Sensors, Networks, SPPCom, SOF), Vancouver, 2016, p. IW1A.3.

[11] S. Li, D. Zhang, J. Zhao, Q. Yang, X. Xiao, S. Hu, L. Wang, M. Li, X. Tang, Y. Qiu, M. Luo, and S. Yu, "Silicon micro-ring tunable laser for coherent optical communication," Optics Express, vol. 24, pp. 6341-6349, 2016/03/21 2016.

[12] N. Li, E. Timurdogan, C. V. Poulton, M. Byrd, E. S. Magden, Z. Su, Purnawirman, G. Leake, D. D. Coolbaugh, D. Vermeulen, and M. R. Watts, "C-band swept wavelength erbium-doped fiber laser with a high-Q tunableinterior-ridge silicon microring cavity," Optics Express, vol. 24, pp. 22741-22748, 2016/10/03 2016.

[13] Y. Liu, K. Wu, N. Li, L. Lan, S. Yoo, X. Wu, P. P. Shum, S. Zeng, and $X$. Tan, "Regenerative Er-doped Fiber Amplifier System for Highrepetition-rate Optical Pulses," Journal of the Optical Society of Korea, vol. 17, pp. 357-361, 2013/10/01 2013.

[14] Y. W. Song, S. A. Havstad, D. Starodubov, Y. Xie, A. E. Willner, and J. Feinberg, "40-nm-wide tunable fiber ring laser with singlemode operation using a highly stretchable FBG," IEEE Photonics Technology Letters, vol. 13, pp. 1167-1169, 2001.

[15] E. H. Bernhardi, H. A. G. M. van Wolferen, L. Agazzi, M. R. H. Khan, C. G. H. Roeloffzen, K. Wörhoff, M. Pollnau, and R. M. de Ridder, "Ultra-narrow-linewidth, single-frequency distributed feedback waveguide laser in Al2O3:Er3+ on silicon," Optics Letters, vol. 35 , pp. 2394-2396, 2010/07/15 2010.

[16] M. Belt, T. Huffman, M. L. Davenport, W. Li, J. S. Barton, and D. J. Blumenthal, "Arrayed narrow linewidth erbium-doped waveguide-distributed feedback lasers on an ultra-low-loss silicon-nitride platform," Optics Letters, vol. 38, pp. 4825-4828, 2013/11/15 2013
[17] M. Belt and D. J. Blumenthal, "High temperature operation of an integrated erbium-doped DBR laser on an ultra-low-loss Si3N4 platform," in Optical Fiber Communications Conference and Exhibition (OFC), 2015, 2015, pp. 1-3.

[18] E. S. Hosseini, Purnawirman, J. D. B. Bradley, J. Sun, G. Leake, T. N. Adam, D. D. Coolbaugh, and M. R. Watts, "CMOS-compatible $75 \mathrm{~mW}$ erbium-doped distributed feedback laser," Optics Letters, vol. 39, pp. 3106-3109, 2014/06/01 2014.

[19] K. van Dalfsen, S. Aravazhi, C. Grivas, S. M. García-Blanco, and M. Pollnau, "Thulium channel waveguide laser in a monoclinic double tungstate with $70 \%$ slope efficiency," Optics Letters, vol. 37, pp. 887-889, 2012/03/01 2012.

[20] K. van Dalfsen, S. Aravazhi, C. Grivas, S. M. García-Blanco, and M Pollnau, "Thulium channel waveguide laser with $1.6 \mathrm{~W}$ of output power and $80 \%$ slope efficiency," Optics Letters, vol. 39, pp. 4380-4383, 2014/08/01 2014

[21] Z. Su, N. Li, E. Salih Magden, M. Byrd, Purnawirman, T. N. Adam, G. Leake, D. Coolbaugh, J. D. B. Bradley, and M. R. Watts, "Ultracompact and low-threshold thulium microcavity lasermonolithically integrated on silicon," Optics Letters, vol. 41 pp. 5708-5711, 2016/12/15 2016

[22] Z. S. J. D. Bradley, E. S. Magden, N. Li, M. Byrd, Purnawirman, T. N. Adam, G. Leake, D. Coolbaugh, M. R. Watts, "1.8- $\mu$ m thulium microlasers integrated on silicon," in SPIE Photonics West, San Francisco, CA, United States, 2016.

[23] N. Li, Purnawirman, J. D. Bradley, G. Singh, E. S. Magden, J. Sun, and M. R. Watts, "Self-pulsing in Erbium-doped fiber laser," in Optoelectronics Global Conference (OGC), 2015, 2015, pp. 1-2.

[24] L. Agazzi, K. Wörhoff, and M. Pollnau, "Energy-TransferUpconversion Models, Their Applicability and Breakdown in the Presence of Spectroscopically Distinct Ion Classes: A Case Study in Amorphous Al2O3:Er3+," The Journal of Physical Chemistry C, vol. 117, pp. 6759-6776, 2013/04/04 2013. 